\title{
Design of $\mathrm{TiO}_{\mathrm{x}} \mathrm{N}_{\mathrm{y}}$ for Coating Technology
}

\author{
Anton Ficai ${ }^{1}$, Denisa Ficai ${ }^{1}$, Bogdan Stefan Vasile ${ }^{1}$, Roxana Doina Trusca ${ }^{1}$, Dragos Gudovan ${ }^{1}$, Ovidiu \\ Oprea $^{1}$, Motelica Ludmila ${ }^{1}$, Ecaterina Andronescu ${ }^{1}$ \\ ${ }^{1}$ Department of Science and Engineering of Oxide Materials and Nanomaterials, Faculty of Applied Chemistry and \\ Materials Science, University Politehnica of Bucharest \\ Polizu Street no 1-7, 011061, Bucharest, Romania \\ anton_ficai81@yahoo.com
}

\section{Extended Abstract}

Titanium dioxide is extensively used for medical and industrial applications including drug delivery, tissue engineering, white pigment, antimicrobial agent, biological active coatings, etc. Based on the literature data, the photocatalytic properties of the $\mathrm{TiO}_{2}$ are strongly dependent on nature and concentration of dopant agents, on morphology and especially size, crystallographic form and so on. $\mathrm{TiO}_{2}$ is also extensively used for developing functional coatings because of the synergism of the surface properties and photocatalytic properties - which induce antimicrobial activity. Even if $\mathrm{TiO}_{2}$ is increasingly used in many applications only limited data are available relating to the modification of $\mathrm{TiO}_{2}$ in order to design it for coating. When titanium dioxide is designed as coating material additional properties are necessary: adhesion on the surface, flexibility, etc. These new characteristics can be easily obtained by modifying the $\mathrm{TiO}_{2}$ by partial substitution of oxygen with nitrogen. Based on the literature data, the content of nitrogen can be between 4 and $70 \%$ with minimal alteration of the desired properties (including biocompatibility). In this context, we proposed three $\mathrm{TiO}_{\mathrm{x}} \mathrm{N}_{\mathrm{y}}$ materials and characterized from the point of view of composition, size and shape and photocatalytic activity. Further works will be necessary them in order to identify the proper deposition conditions onto various surfaces and characterize the coating characteristics (thickness and homogeneity); stability, photocatalytic activity. Based on the preliminary data, the photocatalytic activity is exhibited also in the visible light which is essential in maintaining a clean surface able to selfclean and function during the daily light $[1,2]$. These systems will be possible to use in various applications such as water purification but also can be designed to be applied on glasses to assure the self-cleaning, anti-reflexive surfaces, antifogging, etc. [3, 4]. These materials are also suitable for medical applications and especially for stent technology [5-7]. For medical applications, the cellular response is essential. For stents, it is recommended a low adhesion to cells while, for medical implants, especially bone implants a good adhesion is required. For the environmental applications the non-toxic activity is recommended but the most important characteristic is related to the photocatalytic activity especial in visible light.

Keywords: $\mathrm{TiO}_{\mathrm{x}} \mathrm{N}_{\mathrm{y}}$, Titanium dioxide $\mathrm{TiO}_{2}$, biological active coatings, tissue engineering

\section{Acknowledgements}

The present work was possible due to the EU-funding grant POSCCE-A2O2.2.1-2013-1, Project No. 638/12.03.2014, code SMIS-CSNR 48652. The financial contribution for this research comes from the european project "Titanium Oxynitride Coatings for the Improvement of Biocompatibility and Long-Term Functionality of Cardiovascular Stents: Development of Novel Deposition Technology - TIOXTECH-BIO” Nr. CRT 8/04.04.2016.

\section{References}

[1] J. B. Yoo, H. J. Yoo, H. J. Jung, H. S. Kim, S. Bang, J. Choi, et al, "Titanium oxynitride microspheres with the rocksalt structure for use as visible-light photocatalysts," J Mater Chem A., vol. 4, pp. 869-76, 2016.

[2] M. A. Centeno, M. Paulis, M. Montes, J. A. Odriozola, "Catalytic combustion of volatile organic compounds on gold/titanium oxynitride catalysts," Appl Catal B-Environ., vol. 61, pp. 177-83, 2005. 
[3] X. G. Yang, C. Li, B. J. Yang, W. Wang, Y. T. Qian, "Optical properties of titanium oxynitride nanocrystals synthesized via a thermal liquid-solid metathesis reaction," Chem Phys Lett., vol. 383, pp. 502-6, 2004.

[4] M. Q. Hovish, R. H. Dauskardt, "Optical properties of metal oxynitride thin films grown with atmospheric plasma deposition," J Phys D Appl Phys., vol. 49, 2016.

[5] L. Duta, G. E. Stan, A. C. Popa, M. A. Husanu, S. Moga, M. Socol, et al, "Thickness Influence on In Vitro Biocompatibility of Titanium Nitride Thin Films Synthesized by Pulsed Laser Deposition," Mater., vol. 9, 2016.

[6] M. Hirano, M. Yamane, N. Ohtsu, "Surface characteristics and cell-adhesion performance of titanium treated with direct-current gas plasma comprising nitrogen and oxygen," Appl Surf Sci., vol. 354, pp. 161-7, 2015.

[7] X. L. Cao, T. Sun, Y. H. Yu, "Ti-O-N/Ti composite coating on Ti-6Al-4V: surface characteristics, corrosion properties and cellular responses," J Mater Sci-Mater M., vol. 26, 2015. 